\title{
Assessment of the interoceptive effects of alcohol in rats using short-term training procedures
}

\author{
Joyce Besheera,b,c, Kristen R. Fisher ${ }^{a}$, and Brandon Durant ${ }^{a}$ \\ aBowles Center for Alcohol Studies, University of North Carolina at Chapel Hill, Chapel Hill, North \\ Carolina 27599 \\ ${ }^{b}$ Curriculum in Neurobiology, University of North Carolina at Chapel Hill, Chapel Hill, North \\ Carolina 27599 \\ 'Department of Psychiatry, University of North Carolina at Chapel Hill, Chapel Hill, North Carolina \\ 27599
}

\begin{abstract}
In the present study, we sought to determine whether the interoceptive effects of alcohol $(1 \mathrm{~g} / \mathrm{kg}$, IG) could be assessed using a Pavlovian discrimination method, in which the alcohol drug state sets the occasion for which an environmental stimulus (e.g., light) will be followed by a sucrose reward. This procedure takes advantage of a naturally occurring behavior (i.e., food-seeking) which can be trained rapidly prior to the initiation of discrimination training. Given that the interoceptive effects of alcohol are routinely assessed using operant drug discrimination methods, another group of rats was trained using standard two-lever operant drug discrimination procedures in an effort to compare the Pavlovian procedure to a known behavioral benchmark. The results from this work show that, in addition to operant discrimination procedures, a Pavlovian discrimination task can be used to evaluate the interoceptive effects of alcohol. In addition to the brief behavioral sucrose access training ( 3 days) required prior to the initiation of the Pavlovian discrimination, the alcohol discrimination was acquired relatively rapidly (i.e., 8 training sessions), shortening the overall duration of the experiment. These features of the Pavlovian procedure make it a valuable method by which to assess the interoceptive effects of alcohol if a short experimental time frame is required, such as assessing the interoceptive effects of alcohol during a brief developmental window (e.g., adolescence) or determining the effects of a pretreatment (i.e., chronic stress, chronic drug pretreatment) on the acquisition of the alcohol discrimination. As such, this initial characterization confirms the feasibility of using this Pavlovian discrimination training method as an additional tool by which to assess the interoceptive effects of alcohol, as there may be experimental situations that necessitate short term discrimination training.
\end{abstract}

\section{Keywords}

drug discrimination; Pavlovian; operant; alcoholism; discriminative stimulus; ethanol; drinking; subjective; alcohol

\footnotetext{
(C) 2012 Elsevier Inc. All rights reserved.

Correspondence: Joyce Besheer, Ph.D. (jbesheer@med.unc.edu), Bowles Center for Alcohol Studies, Thurston-Bowles Building; CB\#7178, University of North Carolina at Chapel Hill, Chapel Hill, North Carolina 27599, Voice: 919.843.9478; fax: 919.966.5679.

Publisher's Disclaimer: This is a PDF file of an unedited manuscript that has been accepted for publication. As a service to our customers we are providing this early version of the manuscript. The manuscript will undergo copyediting, typesetting, and review of the resulting proof before it is published in its final citable form. Please note that during the production process errors may be discovered which could affect the content, and all legal disclaimers that apply to the journal pertain.
} 


\section{Introduction}

Psychoactive compounds produce distinct interoceptive effects and these detectable cues are regarded as an important feature of abused substances. An example is the well-known feeling of "drunkenness" or lightheadedness that accompanies alcohol drinking. These interoceptive cues represent a major controlling process that regulates drug-seeking behavior, possibly by supplementing the positive reinforcing effects of drugs (Stolerman, 1992; Stolerman and Jarvis, 1995). The interoceptive effects of drugs can promote drugseeking (Wise et al., 2008), and can provide drug-specific feedback to the organism (Stolerman, 1992). That is, the interoceptive cues that an individual experiences/attends to can prime further drug-taking (e.g., interoceptive effects of a low alcohol dose may prime more drinking) or signal satiety (e.g., interoceptive effects of a higher alcohol dose may limit further drinking). Therefore, examination of the interoceptive effects of drugs of abuse, including alcohol, has important implications for identifying factors that influence pathological behavioral processes, such as excessive drug taking or drinking.

Operant drug discrimination techniques have been widely used to characterize the interoceptive effects of alcohol and other drugs (see (Colpaert, 1999; Glennon and Young, 2011; Porter and Prus, 2009; Stolerman, 1992)). Using these methods, the presence or absence of the interoceptive drug cue guides the operant behavior (e.g., lever press; see Figure 1A). In a two-lever operant procedure, alcohol or vehicle (e.g., water) injections are administered prior to the training session on alternating days. On alcohol training sessions, responses on the left lever (i.e., alcohol-appropriate lever) are reinforced (e.g., presentation of sucrose). On vehicle training sessions, responses on the right lever (water-appropriate lever) are reinforced. For each training session, responses on the alternative levers have no programed consequence. Therefore, the pharmacological effect of alcohol sets the occasion for which responses on the right lever are reinforced, and water sets the occasion for which left lever responses are reinforced. Under these conditions alcohol serves as a discriminative stimulus. These procedures have several advantages including stable and reliable performance over time, which allows for within subject testing, and the ability to utilize response rate measures as indices of drug-induced motor effects. These procedures have proved to be important tools for pharmacological and neurobiological characterization of subjective alcohol effects and abuse liability of novel psychoactive compounds (Becker and Baros, 2006; Besheer et al., 2003; Besheer et al., 2012a; Besheer et al., 2012b; Besheer et al., 2009; Besheer and Hodge, 2005; Cannady et al., 2011; Ginsburg and Lamb, 2005; Grant and Colombo, 1993; Griffin et al., 2012; Gurkovskaya and Winsauer, 2009; Helms and Grant, 2011; Helms et al., 2009; Hodge and Cox, 1998; Hodge et al., 2001; Kostowski and Bienkowski, 1999; Platt and Bano, 2011; Platt et al., 2005; Shelton and Grant, 2002); see also (Ator and Griffiths, 2003; Overton, 1987). However, a requirement of these procedures is that stable operant responding (i.e., lever responses on a specific reinforcement schedule) must be trained prior to the initiation of discrimination training. Further, training subjects to reliably discriminate between the training drug and vehicle can be lengthy (e.g., months), which increases the overall experimental timeframe. Therefore, these procedures have not been suitable for assessing the interoceptive effects of drugs during a brief time period.

Pavlovian conditioning procedures can also be used to train a drug discrimination such that the drug state sets the occasion for when an environmental stimulus (e.g., light) will be followed by a reward (e.g., sucrose; see (Bevins and Murray, 2011)). This procedure has been used to characterize the interoceptive effects of several compounds, such as amphetamine, caffeine, methamphetamine, and nicotine (Murray et al., 2007; Palmatier et al., 2004; Palmatier et al., 2005; Reichel et al., 2007). The Pavlovian procedure used in the present work, incorporates alternating alcohol and vehicle training sessions; however, the 
interoceptive effects of alcohol signal that offset of a brief stimulus (e.g., cue light; conditioned stimulus; CS) is immediately followed by sucrose delivery (unconditioned stimulus; US). Following water administration, the same cue light is presented, but sucrose is not delivered (see Figure 1B). Therefore, the pharmacological effect of alcohol is used to set the occasion upon which the light is paired with sucrose (e.g., feature positive occasion setter). Anticipatory food seeking during the cue light (i.e., head entries into the liquid receptacle; termed "goal-tracking" (Boakes, 1977; Farwell and Ayres, 1979) is the conditioned response (CR) and increases preferentially on alcohol sessions. Similar to the operant procedure, a requirement of the Pavlovian procedure is that the animal engages in a behavior (i.e., approach the sucrose receptacle) to learn the discrimination, and therefore, there are likely convergent learning properties underlying acquisition of alcohol discrimination using the two training procedures. In addition, as with the operant discrimination procedures, Pavlovian discrimination procedures are stable over time, facilitate within subject testing and pharmacological characterization of subjective drug effects (e.g., nicotine, caffeine, amphetamine, methamphetamine) have generally been consistent with the operant literature (Murray et al., 2007; Palmatier et al., 2005; Reichel et al., 2007). Because this procedure takes advantage of a naturally occurring behavior (orienting towards food), the behavior is rapidly acquired which allows for significantly less training time prior to the initiation of discrimination training. In addition, previous work using this procedure has shown rapid acquisition for other drugs (Murray et al., 2007; Palmatier and Bevins, 2008; Palmatier et al., 2004; Palmatier et al., 2005; Reichel et al., 2007; Wilkinson et al., 2009a). Together, these features have the potential to allow for evaluation of the interoceptive effects of alcohol within a short time frame, in contrast to long-term operant procedures, and therefore can be an advantageous tool in certain experimental situations. For example, to assess the interoceptive effects of alcohol during a constrained developmental period (e.g., adolescence), or to determine if the effects of a specific pretreatment (i.e., chronic stress, chronic drug pretreatment) alters sensitivity to alcohol as measured by acquisition of the alcohol discrimination.

To date, interoceptive effects of alcohol have not been investigated using the Pavlovian discrimination procedure described above. Therefore, we sought to determine whether the interoceptive effects of a moderate alcohol dose $(1 \mathrm{~g} / \mathrm{kg})$ could be trained to modulate behavior using the Pavlovian discrimination procedure in rats. Second, in another group of rats alcohol $(1 \mathrm{~g} / \mathrm{kg})$ discrimination was trained using standard two-lever operant drug discrimination procedures in an effort to compare the Pavlovian procedure to a known behavioral benchmark. By definition the two training procedures are inherently different; therefore direct comparison is not entirely appropriate. However, examination of the procedures in parallel allows for a point of comparison given that to date the majority of studies examining the interoceptive effects of alcohol have utilized operant discrimination procedures.

\section{Materials and Methods}

\section{Animals}

Male Long Evans rats (Harlan Sprague Dawley, Indianapolis, IN) weighing 150 - 200 g upon arrival to the colony were individually housed in Plexiglas cages. Twelve rats were used for each training procedure (i.e., Pavlovian; operant); 1 rat in the Pavlovian procedure died unexpectedly early in training, leaving 11 rats in that training condition. Rats were handled and weighed daily for two weeks before training began. Rats were fed approximately 16-25 g of food daily for the duration of the study such that weights maintained at approximately $325-340 \mathrm{~g}$. Water was available continuously in the home cage. The colony room was maintained on a 12-h light/dark cycle and experiments were conducted during the light portion of the cycle. Animals were under continuous care and 
monitoring by veterinary staff from the Division of Laboratory Animal Medicine (DLAM) at UNC-Chapel Hill. All procedures were also carried out in accordance with the NIH Guide to Care and Use of Laboratory Animals and institutional guidelines.

\section{Apparatus}

Pavlovian drug discrimination-Chambers (Med Associates) measuring $31 \times 24 \times 32$ $\mathrm{cm}$ were located within sound attenuating cubicles and equipped with an exhaust fan that provided ventilation and masked external sounds. A cue light was located on one wall of the chamber adjacent to a liquid receptacle equipped with a photobeam detector that was used to detect head entries into the receptacle. Attached to the liquid receptacle was a syringe pump programed to deliver $0.1 \mathrm{ml}$ of $26 \%$ sucrose (w/v) across 1.66 seconds upon activation. This sucrose concentration was selected based on the literature showing that this concentration induced reliable goal-tracking behavior in this task (Murray et al., 2007; Palmatier and Bevins, 2008; Palmatier et al., 2004; Palmatier et al., 2005; Reichel et al., 2007; Wilkinson et al., 2009a).

Operant drug discrimination-Chambers (Med Associates, Georgia, VT) measuring 31 $\times 24 \times 32 \mathrm{~cm}$ were located within sound-attenuating cubicles and equipped with an exhaust fan that provided ventilation and masked external sounds. Two response levers were located on the right wall of each chamber. Responses on the levers activated a liquid dipper centered between the levers that presented a $10 \%$ sucrose (w/v) solution in a $0.1 \mathrm{ml}$ dipper cup for 4 sec during each operation. This sucrose concentration was selected based on our prior work. We did not attempt to equate the concentration to the Pavlovian discrimination procedure given that animals in the operant procedure receive dramatically more sucrose presentations (often $>6 \mathrm{ml}$; see cumulative $10 \%$ sucrose intake in discrimination/self-administration experiments in (Besheer et al., 2012b; Besheer et al., 2006) during a session relative to the Pavlovian procedure in which animals receive 8 sucrose presentations (i.e., $0.8 \mathrm{ml}$ ). Therefore, using $26 \%(\mathrm{w} / \mathrm{v})$ sucrose concentration for the operant procedure may induce satiation during the session and lead to a decrease in lever press behavior. A stimulus light was located above each response lever and was activated each time a reinforcer was delivered. All chambers were interfaced (Med Associates) to a computer programmed to control sessions and record data.

\section{Pavlovian Drug Discrimination Training and Testing Procedures}

Sucrose Access Training-Sucrose access training began with three 50-min sessions providing $26 \%$ sucrose (w/v) randomly across the session to train rats to approach the liquid receptacle (Palmatier et al., 2004). Sucrose $(0.1 \mathrm{ml})$ was delivered randomly throughout the sessions, with probability of sucrose presentation decreasing from the first to the last session. By the last $10 \mathrm{~min}$ of the final session rats received approximately 0.75 sucrose presentations/min.

Acquisition Training-Training sessions were conducted 5 days per week (M-F) during which alcohol $(1 \mathrm{~g} / \mathrm{kg})$ or water was administered IG prior to the start of the sessions. Immediately following the alcohol or water administration the rats were placed in the chambers for 10 min before the session began. During this time no cue lights were illuminated, no sucrose was presented and head entries into the liquid receptacle were not recorded. The 15-min session started after the 10-min delay. During these sessions, the cue light was illuminated for 15-sec. On alcohol sessions, the offset of each of the light presentation was followed by $0.1 \mathrm{ml}$ delivery of sucrose into the liquid receptacle. On water sessions, no sucrose was delivered following the offset of the stimulus light presentations. There were 8 light presentations during both the alcohol and water sessions. The onset of the first light presentation varied from 90-150 s, and the inter-trial intervals (time from CS 
offset to the next CS onset) ranged from 90-210 s. Water and alcohol training days varied on a double alternation schedule (W, W, A, A ...). The training sessions continued until the following criteria were met for both the first and the average discrimination score: the mean of the discrimination scores from the preceding two alcohol sessions had to be greater than the mean of the discrimination scores from the preceding two water sessions by a discrimination score of $\geq 3$ (adapted from (Murray et al., 2007; Palmatier et al., 2004; Palmatier et al., 2005). Testing began once these criteria were met.

Testing-Test sessions were identical to the training sessions except that they were $2 \mathrm{~min}$ in duration (after the 10 min delay), with 1 presentation of the light. The onset of the light presentation varied from 60-105 s and light offset was followed by sucrose delivery. Cumulative alcohol dosing procedures (Besheer et al., 2012a; Besheer et al., 2012b; Besheer et al., 2009; Cannady et al., 2011; Hodge et al., 2001) were used. For example, to determine a cumulative alcohol dose curve $(0.1,0.3,1.0,1.7 \mathrm{~g} / \mathrm{kg}$ ) rats initially received $0.1 \mathrm{~g} / \mathrm{kg}$ alcohol and were placed in the chamber for a testing session. At the conclusion of the session, the rats received a subsequent alcohol administration of $0.2 \mathrm{~g} / \mathrm{kg}$ and another test session. This procedure was repeated with two subsequent administrations of $0.7 \mathrm{~g} / \mathrm{kg}$ alcohol, which are additive to produce the stated dose range. Thus, testing of the entire dose curve was completed in approximately $48 \mathrm{~min}$.

\section{Operant Discrimination Training and Testing Procedures}

Lever press training-Rats experienced two 16-hour sessions to train lever pressing on an fixed-ratio 1 (FR1) schedule of reinforcement (1 session with the left lever, 1 session with the right lever). Next, rats were given 1 hour sessions (M-F) with the presentation of the left and right lever alternating each session. The schedule of reinforcement began at FR2 and gradually increased to FR 10 with the amount of training sessions as follows: 4 sessions at FR2, 2 sessions at FR4, 4 sessions at FR6, 4 sessions at FR8, and 22 sessions at FR10. All rats began discrimination training at the same time once responding on the FR10 schedule was stable $(<10 \%$ daily variation in total number of responses).

Acquisition Training-Training sessions were conducted 5 days per week (M-F) during which alcohol $(1 \mathrm{~g} / \mathrm{kg})$ or water was administered IG prior to the start of the sessions. Immediately following the alcohol or water administration the rats were placed in the chambers and after a 10 min delay the house light was illuminated and both levers were introduced into the chamber signaling the beginning of the 15-min session. Following alcohol administration, completion of 10 responses on the alcohol-appropriate lever resulted in the presentation of the sucrose solution. Similarly, following water administration, completion of 10 responses on the water-appropriate lever resulted in sucrose delivery. During both alcohol and water sessions, responses on the inappropriate lever were recorded but produced no programmed consequences. The left lever was the alcohol-associated lever for half of the rats and the right lever for the other half. Water and alcohol training days varied on a double alternation schedule $(\mathrm{W}, \mathrm{W}, \mathrm{A}, \mathrm{A} \ldots)$. The training sessions continued until the percentage of alcohol- and water-appropriate lever press responses emitted prior to the first reinforcer, and during the entire session was $>80 \%$ for 8 out of 10 consecutive days. These criteria were determined from our previous work and are generally consistent with the drug discrimination literature (Solinas et al., 2006). Once these criteria were met, testing began.

Testing-Test sessions were identical to the training sessions except that they were $2 \mathrm{~min}$ in duration (after the 10 min delay), and completion of an FR10 on either lever resulted in sucrose delivery. Cumulative alcohol dosing procedures were as described for the Pavlovian test. 


\section{Dependent Measures and Data Analysis}

For the Pavlovian discrimination procedure, the number of head entries into the liquid receptacle was recorded in 15 -s intervals. The discrimination score was calculated by subtracting the number of head entries that occurred in the $15 \mathrm{sec}$ before light onset (i.e., pre-CS) from the head entries that occurred during the 15-s light CS (Murray et al., 2007; Palmatier et al., 2004; Palmatier et al., 2005). The first discrimination score (i.e. prior to feedback from sucrose delivery) and the average discrimination score (i.e., includes feedback from sucrose presentation or absence of sucrose) were used as the primary dependent variables. Head entry rate (head entries/min) was analyzed for the entire session. For the substitution test, to confirm that the training dose $(1 \mathrm{~g} / \mathrm{kg})$ induced similar discrimination performance as that during training, a paired $t$-test was used to compare the discrimination score from the $1 \mathrm{~g} / \mathrm{kg}$ alcohol dose at the test to the average of the 2 alcohol sessions prior to testing (Figure $3 \mathrm{~A}$ and $\mathrm{B}$ ). Full substitution for the alcohol training dose was determined when the discrimination score did not differ from the alcohol training dose $(1 \mathrm{~g} / \mathrm{kg})$.

For the operant discrimination procedure, response accuracy was expressed as the percentage of alcohol-appropriate lever presses upon delivery of the first reinforcer (i.e., first FR10 responses; prior to feedback from sucrose presentation) and across the entire session (i.e., average session FR10 responses). Response rate (responses/min) was analyzed for the entire session. For the substitution test, complete substitution for the alcohol stimulus was defined as $\mathbf{2 8 0 \%}$ choice of the alcohol lever upon completion of the first FR10 (Besheer et al., 2009; Solinas et al., 2006).

For acquisition training, two-way repeated measures analysis of variance (ANOVA) were used to analyze accuracy performance (discrimination score; alcohol-appropriate responses) and rate of behavior (response rate; head entry rate). Tukey post-hoc comparisons were used to identify significant interactions and group differences. For the substitution tests, one-way repeated measures ANOVA were used, and Dunnett's tests were used to compare the doses to the alcohol training dose $(1 \mathrm{~g} / \mathrm{kg})$. Significance was declared at $\mathrm{p} \leq 0.05$.

\section{Results}

\section{Pavlovian Drug Discrimination}

The first 10 sessions of acquisition training are shown in Figure 2, as 10 of the 11 rats met the accuracy criteria within an average ( \pm S.E.M.) $17.6 \pm 1.2$ training days (i.e., approximately 8 sessions of each type); 1 rat failed to meet the accuracy criterion after 40 sessions and was excluded from the study. As shown in Figure 2A, after the initial 5 sessions, the first discrimination score increased during alcohol sessions and decreased during the water sessions, demonstrating that the alcohol interoceptive cue was modulating goal-tracking behavior during the light CS. This was confirmed by two-way RM ANOVA that showed a significant main effect of session type (alcohol vs. water; $F(1,9)=12.74, \mathrm{p}<0.006$ ], session $[F(9,81)=5.59, \mathrm{p}<0.001)$ and a significant session type $X$ session interaction $[F(9,81)=7.39$, $\mathrm{p}<0.001]$. The same pattern was observed for the average discrimination score (Figure 2B) as the two way RM ANOVA showed a significant main effect of session type $[F(1,9)=66.04$, $\mathrm{p}<0.001]$, session $[\mathrm{F}(9,81)=13.05, \mathrm{p}<0.001)$ and a significant session type $\mathrm{X}$ session interaction $[\mathrm{F}(9,81)=16.58, \mathrm{p}<0.001]$, and by session 6 discrimination scores on alcohol sessions were significantly greater than the water session $(\mathrm{p}<0.05)$, indicating that the interoceptive effects of alcohol effectively signaled when the light CS would be followed by sucrose. The rate of head entries (i.e., goal-tracking behavior) was significantly elevated during alcohol sessions as compared to water sessions throughout training (Figure 2C). This was confirmed by the two way RM ANOVA that showed a significant main effect of session 
type $[F(1,9)=158.97, p<0.001]$ and a significant interaction $[F(9,81)=6.80, p<0.001]$. Post hoc comparisons showed that head entry rate during alcohol sessions was significantly elevated as compared to water sessions beginning with the initial session $(\mathrm{p}<0.05)$.

After acquisition of the discrimination, the substitution test was conducted. During this test, the light was presented once and light offset was followed by sucrose delivery.

Discrimination scores increased with the alcohol dose $[\mathrm{F}(3,27)=6.27, \mathrm{p}=0.002$; Figure $3 \mathrm{~A}]$, confirming that behavior was under the control of the interoceptive effects of the alcohol training dose $(1 \mathrm{~g} / \mathrm{kg})$. Importantly, the discrimination score at the alcohol training dose $(1 \mathrm{~g} /$ $\mathrm{kg}$ ) was similar to that during training, which confirms feasibility of the cumulative dosing procedures and reliability of performance at the alcohol training dose. There was a significant reduction in the discrimination score at the lowest alcohol dose $(0.1 \mathrm{~g} / \mathrm{kg})$ compared to the training dose $(1 \mathrm{~g} / \mathrm{kg} ; \mathrm{p}<0.05$; Figure $3 \mathrm{~A})$. Therefore, full substitution for the alcohol training dose was observed at the 0.3 and $1.7 \mathrm{~g} / \mathrm{kg}$ doses given that discrimination scores did not differ from $1 \mathrm{~g} / \mathrm{kg}$. Head entry rate also increased with the alcohol dose $[F(3,27)=5.39, p=0.005]$, with significantly lower rate of behavior at the $0.1 \mathrm{~g} /$ $\mathrm{kg}$ alcohol dose than the training dose $(1 \mathrm{~g} / \mathrm{kg}, \mathrm{p}<0.05$; Figure $3 \mathrm{~B})$.

\section{Operant Drug Discrimination}

The first 30 sessions of alcohol discrimination training are illustrated in Figure 4, as 11 of the 12 rats reached the acquisition criteria in an average ( \pm S.E.M.) of $60.1 \pm 1.1$ training days (i.e., approximately 30 sessions of each type). After 150 training days, one rat had not met the acquisition criterion, and was excluded from the study and is not included in any of the data. During the initial 5 training sessions (i.e., 10 days), alcohol-appropriate responding was at chance levels (e.g., $50 \%$ for a two-lever task) prior to delivery of the first reinforcer, but as training progressed this accuracy performance improved (Figure 4A). The two-way RM ANOVA showed a significant main effect of alcohol $[\mathrm{F}(1,10)=442.51, \mathrm{p}<0.001]$, session $[F(29,290)=1.67, p=0.02]$, and a significant interaction $[F(29,290)=9.61, p<0.001]$. Post hoc comparisons showed significantly greater alcohol-appropriate responding on alcohol sessions v. water sessions starting at Session 6 and continuing throughout training $(\mathrm{p}<0.05)$. Examination of the average percent alcohol-appropriate responses across the entire session (Figure 4B), shows high alcohol-appropriate responding following alcohol administration and low alcohol-appropriate responding following water administration as supported by the two-way RM ANOVA that showed a significant main effect of alcohol $[F(1,10)=6575.52, p<0.001]$ and a significant interaction $[F(29,290)=11.31, p<0.001]$. Post hoc comparisons showed significantly greater alcohol-appropriate responding on alcohol sessions v. water sessions starting on Session 1 and continuing throughout training $(\mathrm{p}<0.05)$. Across training sessions, behavior came to be under control of alcohol, as accuracy performance prior to the first reinforcer delivery increased during alcohol sessions (>80\%) and became similar to the total session alcohol-appropriate responses, and alcoholappropriate responses on water sessions decreased $(<20 \%)$. Response rates were stable across the training sessions and similar between alcohol and water sessions (Figure 4C).

After the acquisition criteria were met, a cumulative alcohol substitution curve (Figure 5A) was determined to confirm alcohol control of behavior. A one-way RM ANOVA confirmed that alcohol-appropriate responding increased as a function of alcohol test dose $[F(3,30)=30.22, p<0.001]$, with significantly lower alcohol-appropriate responding at the 0.1 $\mathrm{g} / \mathrm{kg}$ dose relative to the training dose $(1 \mathrm{~g} / \mathrm{kg} ; \mathrm{p}<0.05)$. Full substitution $(>80 \%)$ for the alcohol training dose $(1 \mathrm{~g} / \mathrm{kg}$ ) was confirmed at the two highest alcohol doses ( 1 and $1.7 \mathrm{~g} /$ $\mathrm{kg}$ ). Response rate was not altered by alcohol administration (Figure 5B). These results demonstrate dose-dependent control by alcohol. 


\section{Discussion}

The results from this work show that, in addition to operant discrimination procedures, a Pavlovian discrimination task can be used to evaluate the interoceptive effects of alcohol. In this Pavlovian procedure, the interoceptive effects of alcohol set the occasion for when an environmental stimulus (i.e., light) would be followed by sucrose. In addition to the brief behavioral sucrose access training ( 3 days) required prior to the initiation of the Pavlovian discrimination, the alcohol discrimination was acquired relatively rapidly (i.e., 8 training sessions), as compared to the longer lever press training (20 days on each lever) and number of sessions to meet the acquisition criteria (i.e., 60) in the operant discrimination, thus shortening the overall duration of the experiment. These are attractive features of the Pavlovian procedure which make it a valuable tool by which to assess the interoceptive effects of alcohol during a restricted time frame (e.g., specific stage of development; see later discussion).

The most notable difference between the two procedures is the period of behavioral training prior to the initiation of the discrimination training. That is, in the operant procedure, rats had 20 lever press training sessions on each lever prior to the initiation of discrimination training. This duration of training was determined based on our prior work utilizing the same procedure (Besheer et al., 2012a; Besheer et al., 2012b; Besheer et al., 2009; Besheer and Hodge, 2005; Cannady et al., 2011) and results in robust and stable lever responding behavior on both levers ( $<10 \%$ daily variation in total responses), which is critical in alcohol discrimination studies given that higher alcohol doses can decrease response rates. Clearly, establishing stable lever press behavior and the duration of this training varies between laboratories. Admittedly, a contribution to the lengthy lever press training in the present work and our previous work is that training continues until stable responding at the FR10 schedule has been reached by the entire group, for ease of the daily conduct of the experiment. That is, some rats may show stable FR10 responding after 4 sessions, whereas others may take 12 sessions. Regardless, given that drug discrimination studies typically employ high response requirements (e.g., FR10 utilized in the present work), the necessity to initially establish the lever press behavior, reach the required response schedule, and then obtain stable responding on each lever at the required response schedule before the start of discrimination training, requires more training sessions than establishing goal-tracking behavior in the Pavlovian discrimination. That is, rats in the Pavlovian discrimination were given 3 sucrose access training trials before the initiation of discrimination training (see (Palmatier et al., 2004). This protocol resulted in stable rates of goal-tracking behavior that were maintained throughout the experiment (during alcohol sessions), confirming that this length of sucrose access training was sufficient.

Clearly, direct comparison of the acquisition curves and establishment of an equivalent acquisition criterion is difficult given the inherent differences in the training procedures. For example, the primary dependent variable in the operant discrimination procedures is a relative measure of response allocation (e.g., percentage of responses on the alcoholappropriate lever), as is commonly used. In contrast, the primary dependent variable in the Pavlovian procedure is an absolute difference score of head entries during the 15-sec light (CS) presentation minus the 15-sec before illumination of the light (pre-CS) (Murray et al., 2007; Palmatier et al., 2004; Palmatier et al., 2005; Reichel et al., 2007). Given the low and/ or absence of head entries during the pre-CS periods on both alcohol and water sessions, and the low head entries during water sessions, a relative dependent measure (e.g., head entries during CS/head entries during CS+pre-CS) similar to that used in the operant procedures cannot be determined (i.e., denominator is often 0 for the water sessions). Importantly, if a relative measure of behavior is utilized, the degree of behavioral change would not be captured. For example, if an animal had 1 head entry during the light CS and 0 during the 
pre-CS or 9 head entries during the light and 0 during the pre-CS, both would result in $100 \%$ of goal-tracking behavior during the light CS, but the magnitude of the response is lost, which is critical for interpretation of the behavioral data, especially for substitution tests (i.e., greater goal-tracking during the light CS reflects alcohol-like effects; low/absence of goal-tracking during the light CS occurs on water sessions). For these reasons, the dependent variables used for each procedure were in accordance with the existing literature.

In general, acquisition of the alcohol discrimination appeared to be similar between the two procedures, as evidenced by the pattern of the acquisition curves prior to the first sucrose delivery (i.e., initial discrimination score or initial alcohol-appropriate responses). That is, with training, behavior (goal-tracking or lever selection) is guided by the interoceptive effects of alcohol. A noticeable difference between acquisition of the discrimination in the operant and Pavlovian procedures is evident upon examination of the first vs. average dependent measures. That is, the initial discrimination score in the Pavlovian procedure appears to be fairly reflective of behavior throughout the session (i.e., similar to the average discrimination score; Figure 2A vs. 2B). In contrast, in the operant procedures, the percent of alcohol-appropriate responses prior to the first reinforcer delivery differ dramatically from the average alcohol-appropriate responses (Figure 4A vs. 4B). Again, given the inherent differences in the tasks, this is not to be entirely unexpected. That is, the operant discrimination task requires the animal to make a choice between two levers. Therefore, especially, early on during training, the animal samples each lever to determine which will result in reinforcement (i.e., first alcohol-appropriate response measure), and then maintains responding mostly on that lever throughout the session which results in high accuracy performance (i.e., average alcohol-appropriate response measure). Given that the pattern of discrimination scores (Pavlovian procedure) and alcohol-appropriate responding (operant procedure) are indices of whether behavior is under control of the interoceptive effects of the drug, the similarity between the first and average discrimination scores early on in training in the Pavlovian discrimination, is suggestive of rapid acquisition. Indeed, rats acquired the Pavlovian discrimination in 8 sessions versus 60 sessions in the operant discrimination.

Importantly, both training procedures resulted in reliable stimulus control, as evidenced by dose-dependent alcohol substitution. That is, alcohol-like discrimination performance increased with the alcohol dose. Further, the feasibility of utilizing cumulative testing procedures for the Pavlovian discrimination was demonstrated, as these testing procedures have not been previously utilized. Interestingly, the substitution pattern differed slightly between the two procedures. In the Pavlovian discrimination, alcohol-like effects were observed at the $0.3 \mathrm{~g} / \mathrm{kg}$ dose, whereas in the operant discrimination, low levels of alcoholappropriate responding were observed following this alcohol dose. This finding suggests that the Pavlovian discrimination procedure may be a more sensitive tool for detection of low drug doses than the operant procedures. Indeed, previous work assessing the interoceptive effects of nicotine and amphetamine found substitution at lower doses than reported using operant discrimination procedures (Palmatier et al., 2005). As discussed in that work, these differences may be attributed to different response costs of the procedures. For example, in the Pavlovian procedure there is a relatively small response cost (i.e., approach the receptacle) to gain access to sucrose that is delivered in a predictable manner (i.e., after the light CS) on alcohol sessions. In contrast, in the operant procedure there is a relatively greater response cost (i.e., response requirement on the "correct" lever) to gain access to sucrose on both alcohol and water sessions. Therefore, it is possible that the alcohol interoceptive cue has acquired greater appetitive value during Pavlovian discrimination training, and therefore under these training procedures animals are more sensitive to changes in the pharmacological effects (i.e., lower alcohol dose). Further, in the operant drug discrimination field the schedule of reinforcement has been found to influence 
the shape of the substitution curves. In general, interval reinforcement schedules tend to generate graded (e.g., along a continuum), whereas ratio reinforcement schedules (as used in the present work) tend to generate quantal (e.g., all or none) substitution curves (Colpaert, 1991; Mathis and Emmett-Oglesby, 1990; McMillan et al., 2001; Stolerman, 1991). Therefore, it is possible that the graded alcohol substitution curve in the Pavlovian procedure is partly a consequence of the training procedure. These explanations suggest that sensitivity to the interoceptive effects of alcohol or any test compound may differ depending on the training procedures. Another possible explanation for apparent increased sensitivity to alcohol in the Pavlovian group may be related to the differential alcohol exposure between the two training groups. That is, as a consequence of the shorter discrimination training, rats in the Pavlovian-trained groups received significantly less alcohol exposure than the operant-trained group (i.e., 10 vs. 30 alcohol injections). Therefore, it is possible that the underlying neurobiological processes that modulate sensitivity to alcohol differ as a function of alcohol exposure. It will be interesting for future work to directly test this possibility perhaps by comparing sensitivity to alcohol-like substitutes (i.e., GABAergic compounds, NMDA antagonists) under the two training conditions.

In operant drug discrimination procedures response rate can be used as an index of motor disruption, and changes in accuracy performance (i.e., drug-appropriate responses) are usually independent from response rates (Solinas et al., 2006; Young, 2009). However, a potential drawback to the Pavlovian procedure is the lack of an independent motor index. That is, as a function of the conditioning procedures, rate of behavior (i.e., head entries/min) is high during the alcohol sessions and low during the water sessions. Therefore, if a compound impairs activates general motor activity such that goal-tracking behavior is decreased, it would be difficult to disentangle whether a low discrimination score is the consequence of a general motor deficit or because the compound does not have alcohol-like interoceptive effects. The opposite would be true of a motor activating test compound. Further, a drug-induced change in response rate may be more accurately reflected in procedures in which there is a high response rate requirement such as the operant discrimination procedure, given that high rates of behavior tend to be more resistant to disruption than lower rates of behavior (see (Shahan, 2010). Therefore, in the Pavlovian discrimination measuring general activity simultaneously during the test session (Reichel et al., 2007; Wilkinson et al., 2009b) or in a separate motor assessment (e.g., open field) could be informative to identify whether changes in discrimination performance (goal-tracking) are related to test drug-induced changes in motor behavior.

While other training procedures have been described to assess the interoceptive effects of alcohol (Colombo et al., 1996; Fernandez-Vidal et al., 2003), standard operant drug discrimination techniques continue to be widely used (Becker and Baros, 2006; Besheer et al., 2012a; Besheer et al., 2012b; Cannady et al., 2011; Ginsburg and Lamb, 2005; Griffin et al., 2012; Gurkovskaya and Winsauer, 2009; Helms and Grant, 2011; Hodge et al., 2001; Platt and Bano, 2011; Shelton and Grant, 2002; Stolerman et al., 2011). As we have shown, in addition to operant drug discrimination procedures, behavior can come under the control of the alcohol interoceptive cue using Pavlovian drug discrimination techniques and this can be trained in a relatively brief period of time (e.g., less than three weeks). The ability to rapidly evaluate the interoceptive effects of alcohol has important utility in the alcohol field (as well, as the drug abuse field in general). This method could be used if a short experimental time frame is required, such as evaluation of the interoceptive effects of alcohol during a brief developmental window (e.g., adolescence), or to determine if the effects of a pretreatment (i.e., chronic stress, chronic drug pretreatment) can affect the acquisition of the alcohol discrimination. Further, given the relatively brief experimental time frame, this procedure may be advantageous if conditioning chambers are available for use, but cost is a consideration (i.e., less labor intensive, shorter duration for animal 
maintenance). In addition, given that this procedure takes advantage of a naturally occurring rodent behavior (i.e., goal-tracking), this feature may be advantageous for rapidly evaluating the interoceptive effects of alcohol in mice. While the underlying neuropharmacological and neurobiological targets modulating the interoceptive effects of alcohol have been wellcharacterized using operant procedures (Hodge et al., 2006; Kostowski and Bienkowski, 1999), clearly more investigations will be needed to characterize the alcohol cue using these Pavlovian procedures. In sum, utilization of Pavlovian procedures is an effective method by which to evaluate the interoceptive effects of alcohol. This initial characterization presents an additional tool by which to assess the interoceptive effects of alcohol, as there may be experimental situations in which short-term drug discrimination procedures may be more suitable than longer-term operant drug discrimination procedures.

\section{Acknowledgments}

This work was supported in part by funds from the National Institutes of Health AA019682 and AA016009 (JB) and the Bowles Center for Alcohol Studies. The authors would like to thank Drs. Clyde Hodge, Rick Bevins and Matthew Palmatier for helpful discussions in regards to this manuscript. Also, thanks to Dr. Hodge for the utilization of behavioral equipment.

\section{References}

Ator NA, Griffiths RR. Principles of drug abuse liability assessment in laboratory animals. Drug Alcohol Depend. 2003; 70:S55-72. [PubMed: 12759197]

Becker HC, Baros AM. Effect of duration and pattern of chronic ethanol exposure on tolerance to the discriminative stimulus effects of ethanol in C57BL/6J mice. J Pharmacol Exp Ther. 2006; 319:871-878. [PubMed: 16914560]

Besheer J, Cox AA, Hodge CW. Coregulation of ethanol discrimination by the nucleus accumbens and amygdala. Alcohol Clin Exp Res. 2003; 27:450-456. [PubMed: 12658110]

Besheer J, Fisher KR, Cannady R, Grondin JJ, Hodge CW. Intra-amygdala inhibition of ERK(1/2) potentiates the discriminative stimulus effects of alcohol. Behavioural Brain Res. 2012a; 228:398405.

Besheer J, Fisher KR, Grondin JJ, Cannady R, Hodge CW. The effects of repeated corticosterone exposure on the interoceptive effects of alcohol in rats. Psychopharmacology. 2012b; 220:809-822. [PubMed: 22016195]

Besheer J, Grondin JJ, Salling MC, Spanos M, Stevenson RA, Hodge CW. Interoceptive effects of alcohol require mGlu5 receptor activity in the nucleus accumbens. J Neurosci. 2009; 29:9582-9591. [PubMed: 19641121]

Besheer J, Hodge CW. Pharmacological and anatomical evidence for an interaction between mGluR5and $\operatorname{GABA}(\mathrm{A})$ alpha1-containing receptors in the discriminative stimulus effects of ethanol. Neuropsychopharmacology. 2005; 30:747-757. [PubMed: 15549054]

Besheer J, Stevenson RA, Hodge CW. mGlu5 receptors are involved in the discriminative stimulus effects of self-administered ethanol in rats. Eur J Pharmacol. 2006; 551:71-75. [PubMed: 17026991]

Bevins, RA.; Murray, JE. Internal stimuli generated by abused substances: Role of Pavlovian conditioning and its implications for drug addiction. In: Schachtman, TR.; Reilly, S., editors. Associative learning and conditioning theory: human and non-human applications. London: New York, Oxford University Press; 2011.

Boakes, RA. Performance on learning to associate a stimulus with positive reinforcement. In: Davis, H.; Hurwitz, HMB., editors. Operant-Pavlovian Interactions. Hillsdale, NJ: Lawrence Erlbaum Associates; 1977. p. 67-101.

Cannady R, Grondin JJ, Fisher KR, Hodge CW, Besheer J. Activation of Group II Metabotropic Glutamate Receptors Inhibits the Discriminative Stimulus Effects of Alcohol via Selective Activity Within the Amygdala. Neuropsychopharmacology. 2011 
Colombo G, Agabio R, Balaklievskaia N, Lobina C, Reali R, Fadda F, Gessa GL. T-maze and food reinforcement: an inexpensive drug discrimination procedure. J Neurosci Methods. 1996; 67:8387. [PubMed: 8872872]

Colpaert FC. The discriminative response: an elementary particle of behavior Commentary on Stolerman "Measures of stimulus generalization in drug discrimination experiments". Behav Pharmacol. 1991; 2:283-286. [PubMed: 11224071]

Colpaert FC. Drug discrimination in neurobiology. Pharmacol Biochem Behav. 1999; 64:337-345. [PubMed: 10515310]

Farwell BJ, Ayres JJB. Stimulus-reinforcer and response-reinforcer relations in the control of conditioned appetitive headpoking ("goal-tracking") in rats. Learn Motivation. 1979; 10:295-312.

Fernandez-Vidal JM, Spear NE, Molina JC. Adolescent rats discriminate a mild state of ethanol intoxication likely to act as an appetitive unconditioned stimulus. Alcohol. 2003; 30:45-60. [PubMed: 12878274]

Ginsburg BC, Lamb RJ. Alphaxalone and epiallopregnanolone in rats trained to discriminate ethanol. Alcohol Clin Exp Res. 2005; 29:1621-1629. [PubMed: 16205362]

Glennon, RA.; Young, R. An Introduction to drug discrimination. In: Glennon, RA.; Young, R., editors. Drug discrimination: applications to medicinal chemistry and drug studies. Hoboken, New Jersey: Wiley; 2011. p. 3-14.

Grant KA, Colombo G. Pharmacological analysis of the mixed discriminative stimulus effects of ethanol. Alcohol Alcohol Suppl. 1993; 2:445-449. [PubMed: 7748336]

Griffin WC 3rd, Nguyen SA, Deleon CP, Middaugh LD. Effects of vigabatrin, an irreversible GABA transaminase inhibitor, on ethanol reinforcement and ethanol discriminative stimuli in mice. Behav Pharmacol. 2012; 23:178-190. [PubMed: 22336593]

Gurkovskaya OV, Winsauer PJ. Discriminative stimulus effects of ethanol, pregnanolone, and dehydroepiandrosterone (DHEA) in rats administered ethanol or saline as adolescents. Pharmacol Biochem Behav. 2009; 93:82-90. [PubMed: 19393687]

Helms CM, Grant KA. The effect of age on the discriminative stimulus effects of ethanol and its GABA(A) receptor mediation in cynomolgus monkeys. Psychopharmacology. 2011; 216:333343. [PubMed: 21340471]

Helms CM, Rogers LS, Grant KA. Antagonism of the ethanol-like discriminative stimulus effects of ethanol, pentobarbital, and midazolam in cynomolgus monkeys reveals involvement of specific GABA(A) receptor subtypes. J Pharmacol Exp Ther. 2009; 331:142-152. [PubMed: 19641166]

Hodge CW, Cox AA. The discriminative stimulus effects of ethanol are mediated by NMDA and GABA(A) receptors in specific limbic brain regions. Psychopharmacology (Berl). 1998; 139:95107. [PubMed: 9768547]

Hodge CW, Cox AA, Bratt AM, Camarini R, Iller K, Kelley SP, Mehmert KK, Nannini MA, Olive MF. The discriminative stimulus properties of self-administered ethanol are mediated by GABA(A) and NMDA receptors in rats. Psychopharmacology (Berl). 2001; 154:13-22. [PubMed: 11292001]

Hodge CW, Grant KA, Becker HC, Besheer J, Crissman AM, Platt DM, Shannon EE, Shelton KL. Understanding how the brain perceives alcohol: neurobiological basis of ethanol discrimination. Alcohol Clin Exp Res. 2006; 30:203-213. [PubMed: 16441269]

Kostowski W, Bienkowski P. Discriminative stimulus effects of ethanol: neuropharmacological characterization. Alcohol. 1999; 17:63-80. [PubMed: 9895039]

Mathis DA, Emmett-Oglesby MW. Quantal vs. graded generalization in drug discrimination: measuring a graded response. J Neurosci Methods. 1990; 31:23-33. [PubMed: 2407907]

McMillan DE, Hardwick WC, Li M. Discrimination of pentobarbital doses and drug mixtures under fixed-ratio and fixed-interval reinforcement schedules. Behav Pharmacol. 2001; 12:195-208. [PubMed: 11485056]

Murray JE, Li C, Palmatier MI, Bevins RA. The interoceptive Pavlovian stimulus effects of caffeine. Pharmacol Biochem Behav. 2007; 86:838-846. [PubMed: 17477964]

Overton, DA. Applications and Limitations of the Drug Discrimination Method for the Study of Drug Abuse. In: Bozarth, MA., editor. Methods of assessing the reinforcing properties of abused drugs. New York: Springer-Verlag; 1987. p. 291-340. 
Palmatier MI, Bevins RA. Occasion setting by drug states: Functional equivalence following similar training history. Behav Brain Res. 2008; 195:260-270. [PubMed: 18845189]

Palmatier MI, Peterson JL, Wilkinson JL, Bevins RA. Nicotine serves as a feature-positive modulator of Pavlovian appetitive conditioning in rats. Behav Pharmacol. 2004; 15:183-194. [PubMed: 15187576]

Palmatier MI, Wilkinson JL, Metschke DM, Bevins RA. Stimulus properties of nicotine, amphetamine, and chlordiazepoxide as positive features in a pavlovian appetitive discrimination task in rats. Neuropsychopharmacology. 2005; 30:731-741. [PubMed: 15592350]

Platt DM, Bano KM. Opioid receptors and the discriminative stimulus effects of ethanol in squirrel monkeys: Mu and delta opioid receptor mechanisms. EurJ Pharmacol. 2011; 650:233-239. [PubMed: 20940013]

Platt DM, Duggan A, Spealman RD, Cook JM, Li X, Yin W, Rowlett JK. Contribution of alpha 1GABAA and alpha 5GABAA receptor subtypes to the discriminative stimulus effects of ethanol in squirrel monkeys. J Pharmacol Exp Ther. 2005; 313:658-667. [PubMed: 15650112]

Porter JH, Prus AJ. Drug discrimination: 30 years of progress. Psychopharmacology. 2009; 203:189_ 191. [PubMed: 19225764]

Reichel CM, Wilkinson JL, Bevins RA. Methamphetamine functions as a positive and negative drug feature in a Pavlovian appetitive discrimination task. Behav Pharmacol. 2007; 18:755-765. [PubMed: 17989513]

Shahan TA. Conditioned reinforcement and response strength. J Exp Anal Behav. 2010; 93:269-289. [PubMed: 20885815]

Shelton KL, Grant KA. Discriminative stimulus effects of ethanol in C57BL/6J and DBA/2J inbred mice. Alcohol Clin Exp Res. 2002; 26:747-757. [PubMed: 12068241]

Solinas M, Panlilio LV, Justinova Z, Yasar S, Goldberg SR. Using drug-discrimination techniques to study the abuse-related effects of psychoactive drugs in rats. Nat Protoc. 2006; 1:1194-1206. [PubMed: 17406402]

Stolerman I. Drugs of abuse: behavioural principles, methods and terms. Trends Pharmacological Sci. 1992; 13:170-176.

Stolerman IP. Measures of stimulus generalization in drug discrimination experiments. Behav Pharmacol. 1991; 2:265-282. [PubMed: 11224070]

Stolerman IP, Childs E, Ford MM, Grant KA. Role of training dose in drug discrimination: a review. Behav Pharmacol. 2011; 22:415-429. [PubMed: 21808191]

Stolerman IP, Jarvis MJ. The scientific case that nicotine is addictive. Psychopharmacology. 1995; 117:2-10. discussion 14-20. [PubMed: 7724697]

Wilkinson JL, Li C, Bevins RA. Pavlovian drug discrimination with bupropion as a feature positive occasion setter: substitution by methamphetamine and nicotine, but not cocaine. Addict Biol. 2009a; 14:165-173. [PubMed: 19076926]

Wilkinson JL, Li C, Bevins RA. Pavlovian drug discrimination with bupropion as a feature positive occasion setter: substitution by methamphetamine and nicotine, but not cocaine. Addict Biol. 2009b; 14:165-173. [PubMed: 19076926]

Wise RA, Wang B, You ZB. Cocaine serves as a peripheral interoceptive conditioned stimulus for central glutamate and dopamine release. PLoS One. 2008; 3:e2846. [PubMed: 18682722]

Young, R. Drug Discrimination. In: Buccafusco, JJ., editor. Methods of Behavior Analysis in Neuroscience. Boca Raton (FL): CRC Press; 2009. 
A

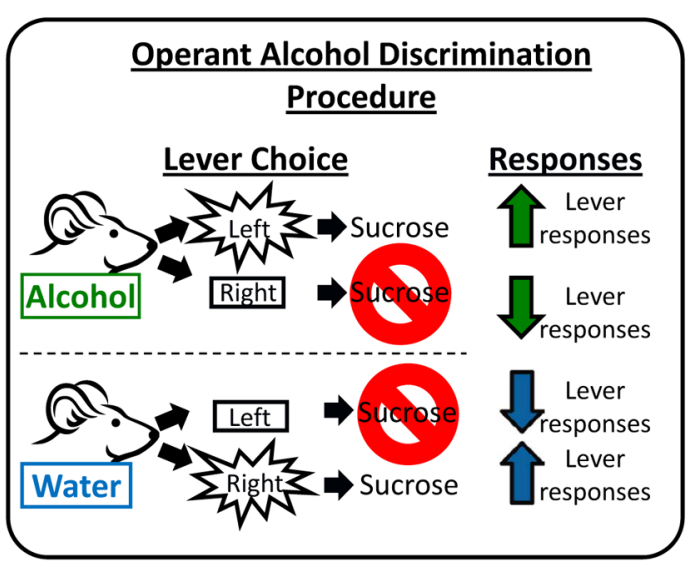

B

\section{Pavlovian Alcohol Discrimination} Procedure

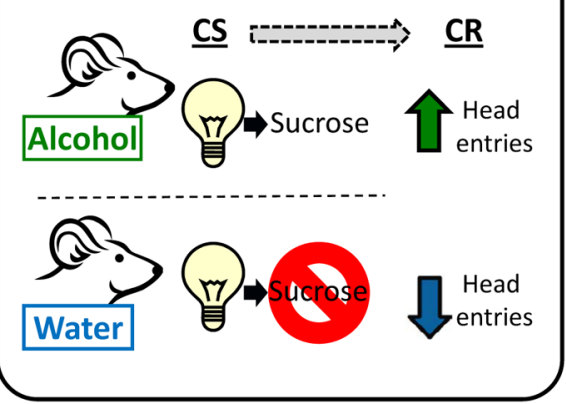

Figure 1. Illustration of drug discrimination procedures

(A) In the operant drug discrimination procedure, on alcohol training sessions, alcohol sets the occasion for which responses on the left lever result in sucrose delivery; responses on right lever have no consequences. This leads to an increase in alcohol-appropriate (i.e., left) lever responses. On water training sessions, responses on the right lever result in sucrose delivery; responses on the left lever have no consequences. This lead to an increase in waterappropriate (i.e., right) lever responses. (B) In the Pavlovian drug discrimination procedure, on alcohol sessions, alcohol sets the occasion for which the offset of a light (conditioned stimulus; CS) will be followed by sucrose delivery, which leads to an increase in head entries (conditioned response; CR) during the light. On water sessions, no sucrose is delivered following light offset, which lead to a decrease in head entries during the light CS. 

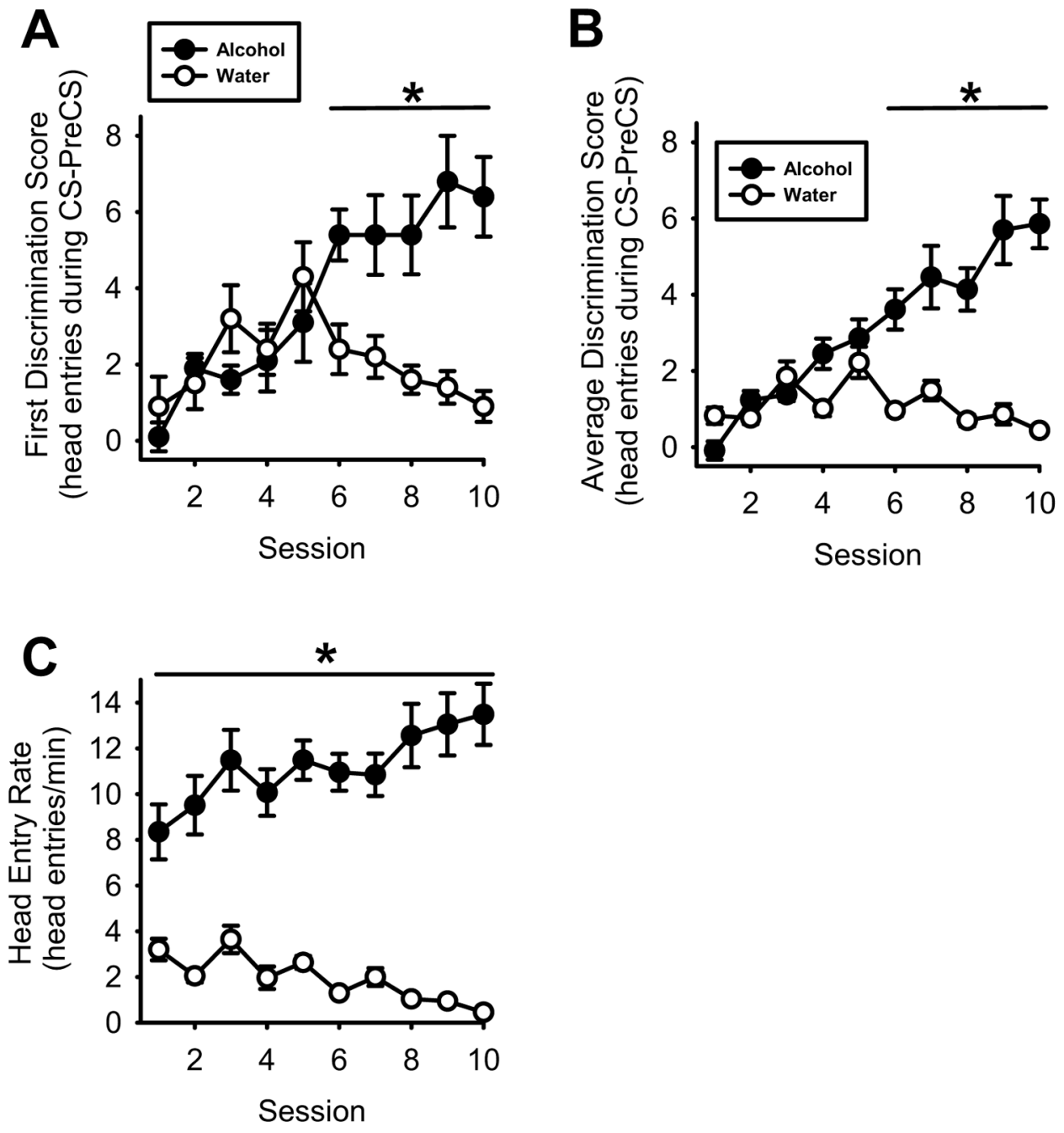

Figure 2. Acquisition of the alcohol discrimination using Pavlovian procedures (A) Across sessions, mean ( \pm S.E.M.) discrimination scores during the first light CS presentation (e.g., prior to the first sucrose delivery) during alcohol ( $1 \mathrm{~g} / \mathrm{kg}$, IG) sessions increased. By session 6, discrimination scores on alcohol sessions were significantly higher than water sessions. (B) Similar to the first discrimination score, the average discrimination score for the entire session increased during alcohol sessions, and was significantly greater than water sessions by session 6. (C) Mean ( \pm S.E.M.) head entry rate (head entries/min) during alcohol sessions was significantly greater than water sessions throughout training. * denotes significantly different from alcohol $(\mathrm{p}<0.05)$. 


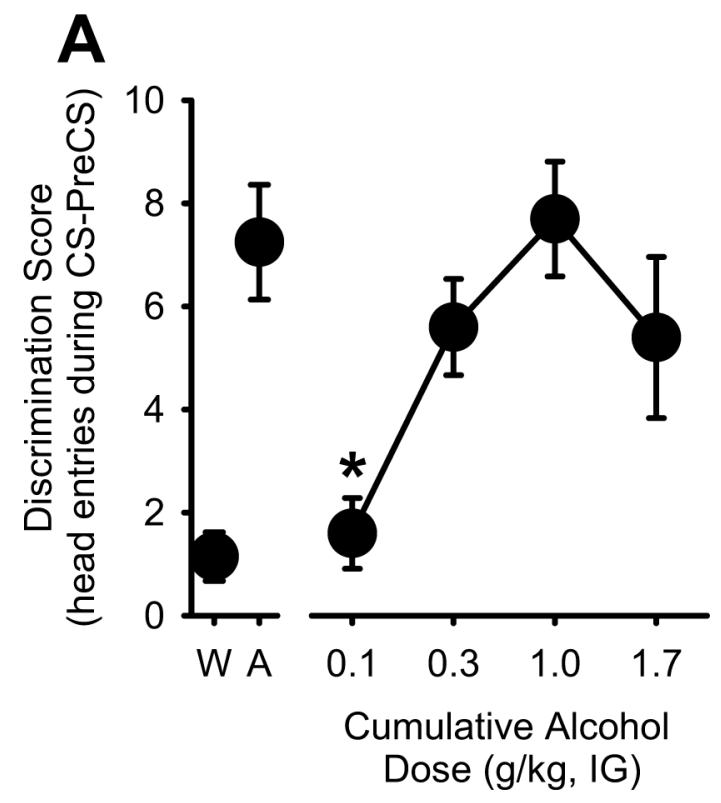

B

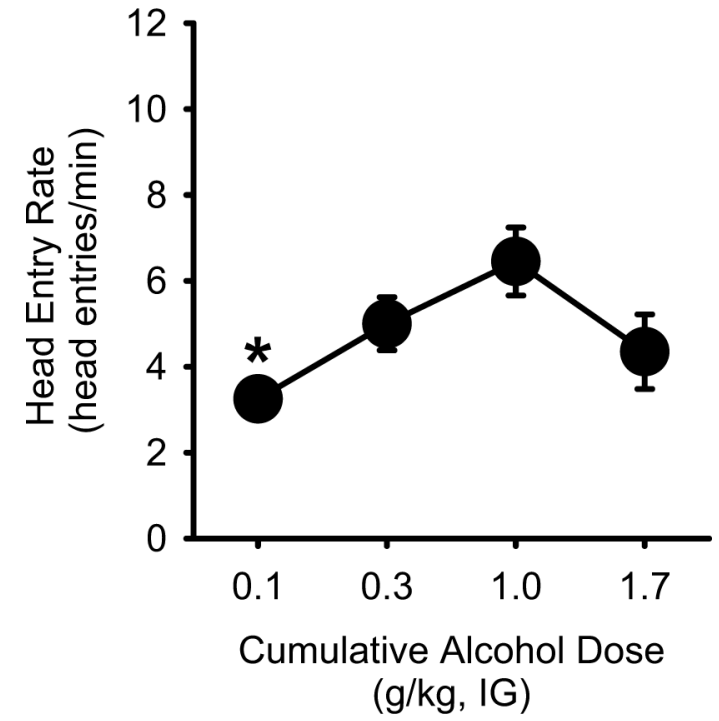

Figure 3. Alcohol substitution using Pavlovian procedures

(A) Average ( \pm S.E.M.) discrimination scores from the two alcohol and water sessions that preceded testing are represented at the left of axis break. Dose-dependent substitution for the $1 \mathrm{~g} / \mathrm{kg}$ alcohol dose was observed, demonstrating that the training procedures established reliable control of goal-tracking behavior. (B) Mean ( \pm S.E.M.) head entry rate (head entries/ min) was significantly lower at the lowest alcohol dose $(0.1 \mathrm{~g} / \mathrm{kg})$. * denotes significantly different from the $1 \mathrm{~g} / \mathrm{kg}$ alcohol training dose $(\mathrm{p}<0.05)$. 

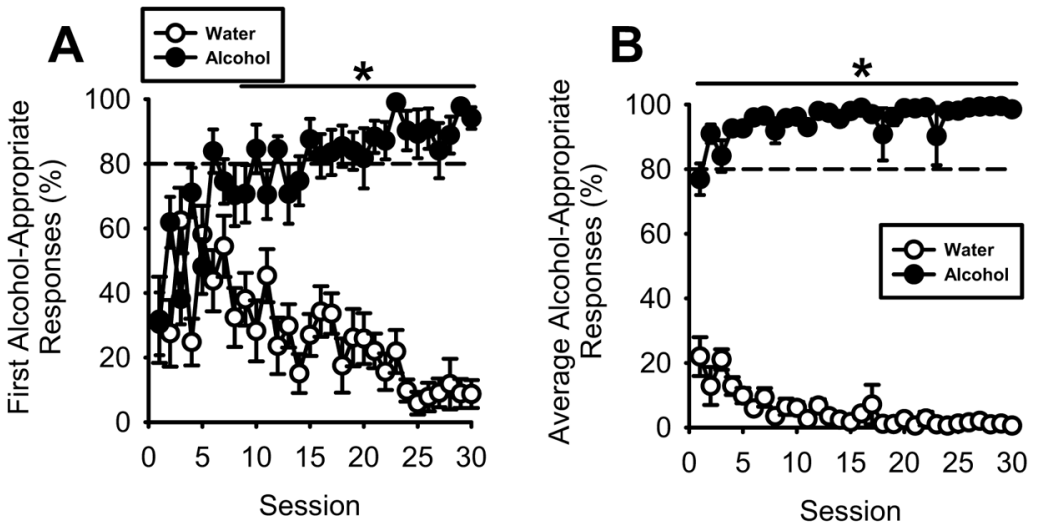

C

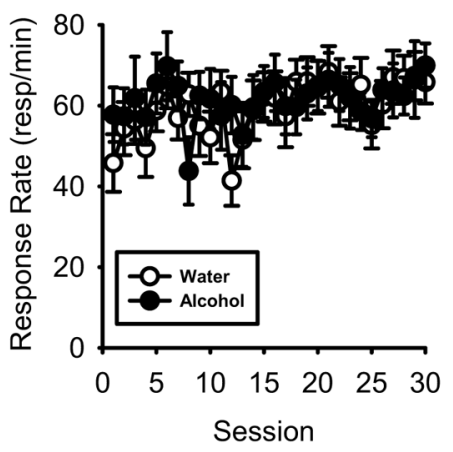

Figure 4. Acquisition of the alcohol discrimination using operant procedures

(A) Across training sessions, alcohol-appropriate responses after completion of the first FR10 requirement (i.e., prior to the first sucrose delivery) increased during alcohol $(1 \mathrm{~g} / \mathrm{kg}$, IG) sessions and decreased during water sessions. By session 6, alcohol-appropriate responses were significantly higher during alcohol sessions than water sessions. By session 15 alcohol-appropriate responses were consistently $\geq 80 \%$ after alcohol administration and consistently $\mathfrak{2} 0 \%$ after water administration by session 24. (B) Average alcohol-appropriate responses during alcohol sessions were significantly greater than water sessions throughout training, which reflects, in part, influence of reinforcer delivery. (C) Response rate (responses/min) did not vary between alcohol and water sessions. 

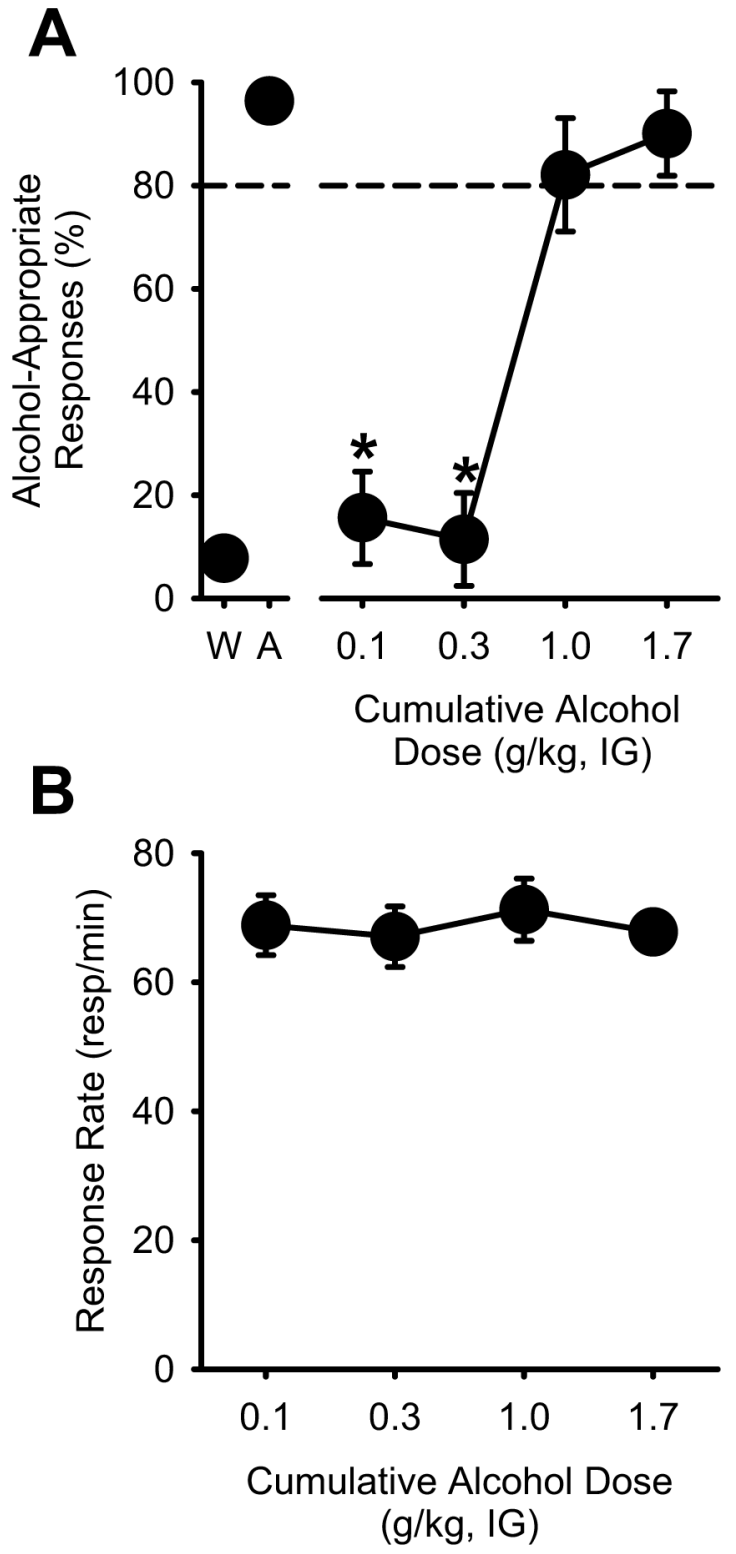

Figure 5. Alcohol substitution using operant procedures

(A) Average ( \pm S.E.M.) alcohol-appropriate responses from the two alcohol and water sessions that preceded testing are represented at the left of axis break. Dose-dependent substitution for the $1 \mathrm{~g} / \mathrm{kg}$ alcohol dose was observed, demonstrating that the training procedures established reliable control of behavior. (B) Response rate (responses/min) did not vary by alcohol dose. * denotes significantly different from the $1 \mathrm{~g} / \mathrm{kg}$ alcohol training dose $(\mathrm{p}<0.05)$. 